\title{
Pathways of activated escape in periodically modulated systems
}

\author{
D. Ryvkine and M. I. Dykman \\ Department of Physics and Astronomy, Michigan State University
}

(Dated: August 7, 2018)

\begin{abstract}
We investigate dynamics of activated escape in periodically modulated systems. The trajectories followed in escape form diffusion broadened tubes, which are periodically repeated in time. We show that these tubes can be directly observed and find their shape. Quantitatively, the tubes are characterized by the distribution of trajectories that, after escape, pass through a given point in phase space for a given modulation phase. This distribution may display several peaks separated by the modulation period. Analytical results agree with the results of simulations of a model Brownian particle in a modulated potential.

PACS numbers: 05.40.-a, 02.50.-r, 05.70.Ln, 77.80.Fm
\end{abstract}

\section{INTRODUCTION}

The problem of noise-activated escape in periodically modulated systems is of interest to many areas of physics and applications, from Josephson junctions [1, 2, 3] and nano- and micromechanical systems [4, 5] to epidemics [6]. The theory of activated escape should answer two closely related questions: what is the escape rate and how does the system move during escape? Fluctuational trajectories leading to escape or interstate switching are of significant interest and have been extensively studied in recent years using various numerical techniques, see Refs. 6, 7, 8, 9 and papers cited therein. Even though escape is a random event, the probabilities of following different paths are strongly different. Therefore most likely the system follows a certain pathway, i.e., its trajectory is close to the most probable escape path (MPEP), see Ref. 10 and references therein.

The distribution of escape trajectories can be conveniently characterized by the prehistory probability distribution (PPD), a quantity accessible to direct experimental measurements. It is obtained by recording trajectories of the system that lead to escape and superimposing the trajectories that, after the system has escaped, pass through a small vicinity of a point $q_{f}$ (for a certain modulation phase, in the case of a periodically modulated system). The point $q_{f}$ is chosen in the phase space sufficiently far behind the boundary of the basin of attraction to the initially occupied metastable state. Formally, the $\operatorname{PPD} p_{h}\left(q, t \mid q_{f}, t_{f}\right)$ is the probability density for the system to have passed through a point $q$ at an instant $t$ provided it had been fluctuating about the metastable state for a long time and passed $q_{f}$ at a later time $t_{f}$, $t_{f}>t[11]$.

The PPD $p_{h}\left(q, t \mid q_{f}, t_{f}\right)$ should peak at $q$ lying on the optimal fluctuational path that leads to $q_{f}$. Therefore it "maps out" optimal paths. For stationary systems this has been directly confirmed by extensive simulations 12 , 13, 14] and also in laser experiments 15. In such systems escape can occur at any time, with equal probability, therefore the tube of paths around the optimal escape path is broad.

In the present paper we study escape pathways in pe- riodically modulated systems. Modulation synchronizes escape events. This can be easily understood for escape from a slowly modulated potential well. Here, escape is most likely to occur when the instantaneous potential barrier $\Delta U(t)$ is at its lowest, once per period, cf. Fig. 1(a). If the modulation amplitude of $\Delta U(t)$ significantly exceeds the noise intensity $D\left(D=k_{B} T\right.$ for thermal noise), escape events are synchronized very strongly. Strong synchronization persists even where the modulation is not slow and the adiabatic picture in which the escape rate is determined by the instantaneous barrier height does not apply [16, 17, 18, 19, 20]. As a result of escape synchronization, there is one MPEP per period.

In turn, as we show, the prehistory probability distribution displays a sharp narrow peak as a function of $q$ for a given $t$. This peak lies on the MPEP. Respectively, in $(q, t)$ space the PPD displays a narrow ridge centered at the MPEP, cf. Fig. 2 Moreover, the PPD may have several narrow peaks [ridges in $(q, t)$ space]. Their width gives the width of the distribution of escape trajectories. It is determined by the typical diffusion length $l_{D}=\left(2 D t_{\mathrm{r}}\right)^{1 / 2}$, where $t_{\mathrm{r}}$ is the relaxation time of the system. This is qualitatively different from the shape of the PPD in stationary systems [15]. Of course, in modulated systems along with the final point $q_{f}$ through which the system passes one should fix the modulation phase when the passage happens. It is given by the passage time $t_{f}\left(\bmod \tau_{F}\right)$, where $\tau_{F}$ is the modulation period.

The occurrence of a narrow peak of the escape trajectories distribution can be understood from the qualitative picture of motion in escape. This picture is sketched in Fig. 1for a system with one dynamical variable $q$. Escape from a static potential well corresponds to going over the barrier top $q_{b}$ from the vicinity of the potential minimum $q_{a}$, see Fig. 1(a). Similarly, a modulated system escapes when it goes over the periodic basin boundary $q_{b}(t)$ from a periodic metastable state $q_{a}(t)$, see Fig. If b) (here and below we assume that the noise correlation time is small; see Ref. 21 for a more general case). Let us suppose that the escaped particle is found at time $t_{f}$ at a point $q_{f}$ sufficiently far behind $q_{b}(t)$. A typical trajectory to this point displays four distinct sections with different types of motion shown schematically by letters A through D. 

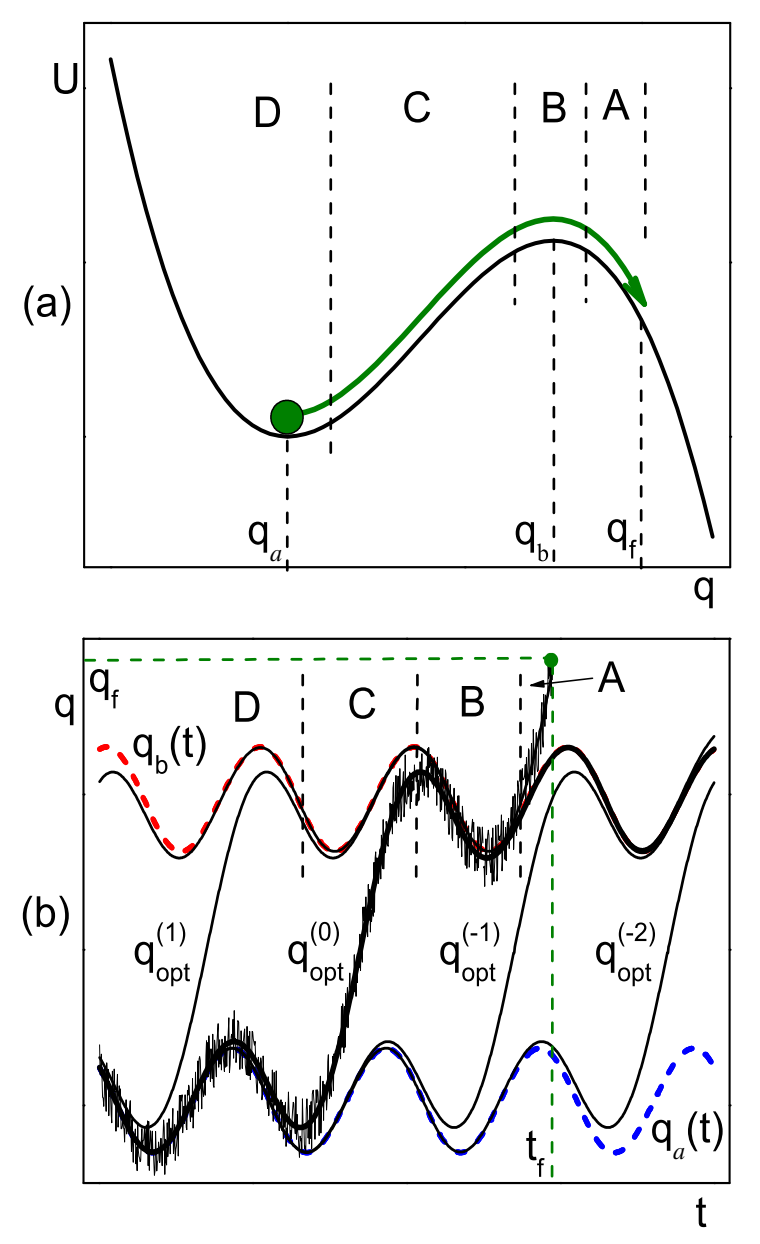

FIG. 1: (Color online) Activated motion leading to detection of an escaped particle at point $q_{f}$ at time $t_{f}$ (schematically). Panels (a) and (b) illustrate escape from a static and a periodically modulated potential well, respectively. In the latter case $q_{a}(t)$ and $q_{b}(t)$ are the periodic stable state and the basin boundary. The trajectories $q_{\mathrm{opt}}^{(n)}(t)(n=1, \ldots,-2)$ are the periodically repeated most probable escape paths. The four major stages of motion $\mathrm{A}, \mathrm{B}, \mathrm{C}$, and $\mathrm{D}$ on the way to $q_{f}$ are discussed in the text.

We discuss the motion backward in time from $t_{f}$. Immediately adjacent to $q_{f}$ is section A of the trajectory in Fig. [1 Here, for small noise intensity the system moves close to the noise-free trajectory from the vicinity of $q_{b}(t)$ to $q_{f}$. In the case of a static potential this corresponds to sliding down the potential slope from $q_{b}$ to $q_{f}$. Section B corresponds to diffusion in a region of width $\sim l_{D}$ around $q_{b}(t)$. In this region noise-free motion with respect to $q_{b}(t)$ is slow (in the case of a static potential the potential is locally flat at $\left.q_{b}\right)$. Section C corresponds to motion from the attractor to the basin boundary. The respective trajectory is close to the most probable escape path. This motion is a result of the large fluctuation that has led to escape detected at $q_{f}$. For strongly synchronized escape, there is one MPEP per modulation period, as shown in
Fig. 1(b). The MPEP approaches $q_{b}(t)$ asymptotically as $t \rightarrow \infty$. If the system was observed behind $q_{b}\left(t_{f}\right)$ at time $t_{f}$, it has most likely arrived to the vicinity of $q_{b}(t)$ along the MPEP that approached $q_{b}(t)$ before $t_{f}$, but not too much in advance, as shown in Fig. 1b). Finally in the region $\mathrm{D}$ well before $t_{f}$ the system was fluctuating about the attractor.

The above picture suggests that the PPD will peak on the trajectory singled out in Fig. 1. It also explains why the PPD peak along $q$ axis should be narrow: in contrast to stationary systems, which can arrive to the vicinity of $q_{b}$ at any time, periodically modulated systems approach $q_{b}(t)$ only once per period, and the tube of escape trajectories is narrow. We note that, by changing $q_{f}, t_{f}$, we can switch between neighboring MPEP's, and therefore there is a possibility for the PPD to have two and potentially even more peaks inside the attraction basin.

As mentioned above, we are interested in the regime where escape events are strongly synchronized by the modulation. It means that the probability density to find the escaped system at a point $q_{f}$ behind the basin boundary, cf. Fig. 11 displays sharp peaks as a function of the observation time $t_{f}$. These peaks are periodically repeated in time. They were studied in Ref. 20. We will analyze the PPD for the time $t_{f}$ close to the maximum of the escape probability peak. This choice is justified, because the corresponding PPD characterizes the most probable escape trajectories. Otherwise the PPD would be formed by trajectories with exponentially smaller probabilities that are very rarely followed in escape.

Interestingly, the condition that the tubes of escape trajectories be narrow in time and space requires that the modulation frequency $\omega_{F}=2 \pi / \tau_{F}$ lie within a range limited both from below and from above. To understand the lower limit we note that, as mentioned above, for slow modulation escape occurs every period around the time $t_{\mathrm{m}}+k \tau_{F}$ where the height of the instantaneous potential barrier $\Delta U(t)$ is at its minimum $(k=0, \pm 1, \ldots)$. The typical width of the time window for escape $\Delta t$ is given by the condition $\left|\Delta U\left(t_{\mathrm{m}} \pm \Delta t\right)-\Delta U\left(t_{\mathrm{m}}\right)\right| \lesssim D$, which leads to $\Delta t=\left[D / \Delta \ddot{U}\left(t_{\mathrm{m}}\right)\right]^{1 / 2} \sim \omega_{F}^{-1}\left[D / \Delta U\left(t_{\mathrm{m}}\right)\right]^{1 / 2}$. If $\Delta t$ exceeds the typical duration of motion in escape $t_{\mathrm{r}} \ln \left[\Delta U\left(t_{\mathrm{m}}\right) / D\right]$, the PPD has the same shape as if the system were escaping out of a stationary potential well of height $\Delta U\left(t_{\mathrm{m}}\right)$. In this case inside the attraction basin the ridge of the PPD in $(q, t)$ space is broad and asymmetric, its width along $q$ axis is independent of the noise intensity [15].

In the opposite limit of high-frequency modulation, $\omega_{F} t_{\mathrm{r}} \gg 1$, escape of an overdamped system is not synchronized. The dynamics is characterized by the coordinates averaged over modulation period. The PPD as a function of such coordinates is described by the theory for stationary systems [15], and the PPD peak inside the attraction basin is broad.

In the intermediate range of frequencies not only are the PPD peaks narrow but, as mentioned above, the PPD 


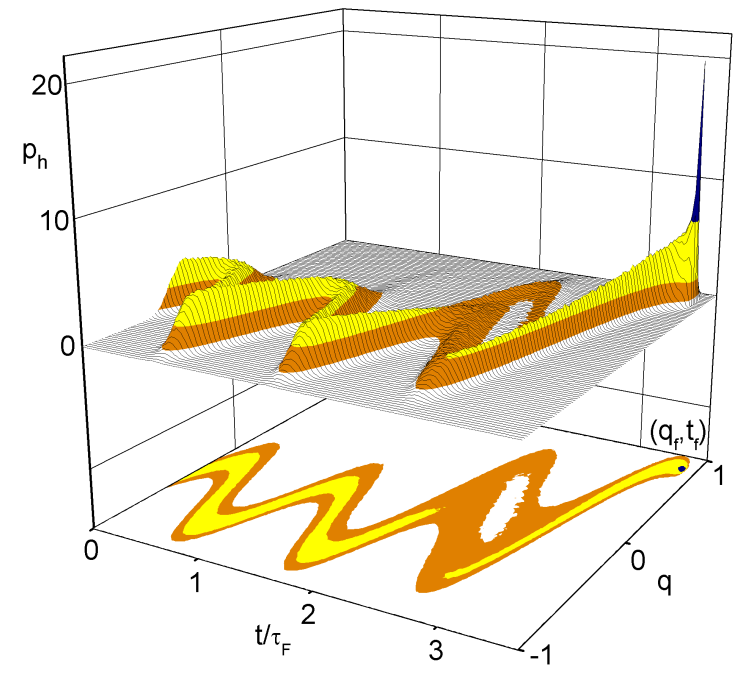

FIG. 2: (Color online) The prehistory probability density (PPD) $p_{h}\left(q, t \mid q_{f}, t_{f}\right)$ and its contour plot for a noise-driven overdamped system with equation of motion $\dot{q}=q^{2}-0.25+$ $A \cos \omega_{F} t+f(t)$, where $f(t)$ is white noise of intensity $D$. The parameters are $A=0.7, \omega_{F}=2, D=0.01, q_{f}=0.8$, $t_{f}=\left(\tau_{F} / 2\right)\left(\bmod \tau_{F}\right)$, where $\tau_{F}=2 \pi / \omega_{F}$ is the modulation period. The shadowing (color code on line) corresponds to the 4 regions of the height of the distribution separated by the values $p_{h}=0.5,2,7$.

as a function of $q$ for given $t$ (and also of $t$ for given q) may display several peaks inside the attraction basin. This happens because the motion of the system near the basin boundary is slow. Therefore if the system is observed behind the boundary $q_{b}(t)$ at a given time $t_{f}$, it could have arrived to the boundary along one of a few periodically repeated optimal escape paths, fluctuated about $q_{b}(t)$ for some time, and then made a transition to $\left(q_{f}, t_{f}\right)$ over time $\sim t_{\mathrm{r}}$.

The multiple-peak structure of the PPD is a specific feature of periodically modulated systems far from equilibrium, i.e. away from both the adiabatic limit of slow modulation and the limit of fast modulation. It is illustrated in Fig. 2 for a model system. Two ridges of the PPD inside the attraction basin are clearly resolved in this figure. Their shape is well described by the asymptotic theory developed in this paper.

In Sec. II below we discuss the dynamics of a periodically modulated system and the equations for the most probable escape path. In Sec. III we obtain a general expression for the PPD near the basin boundary and relate it to the distribution that describes the quasiperiodic current away from the attraction basin, which gives the escape rate. In Sec. IV it is shown that the PPD is simplified in the adiabatic limit where the modulation period is small compared to the relaxation time. Two types of behavior may be displayed near $q_{b}(t)$ in this case, de- pending on the ratio of the two small parameters that characterize the dynamics. The analysis is extended to the case of nonadiabatic driving in Sec. V. In Sec. VI we obtain the central result of the paper, the PPD inside the attraction basin. We show that it has the shape of a sum of diffusion-broadened Gaussian peaks centered at the periodically repeated most probable escape paths. In Sec. VII we describe the results of simulations of a model system and compare them to the analytical results. Sec. VIII provides a brief summary of the results.

\section{ESCAPE OF A PERIODICALLY MODULATED SYSTEM}

We investigate the prehistory probability distribution for an overdamped system characterized by one dynamical variable $q$. The system is in a periodically modulated potential $U(q, t)=U\left(q, t+\tau_{F}\right)$. Fluctuations are induced by an external noise $f(t)$. The motion is described by the Langevin equation

$$
\dot{q}=K(q, t)+f(t) .
$$

Here, $K(q, t) \equiv \partial_{q} U(q, t)$ is the regular periodic force. We consider the simplest case where $f(t)$ is zero-mean white Gaussian noise with correlator $\left\langle f(t) f\left(t^{\prime}\right)\right\rangle=2 D \delta\left(t-t^{\prime}\right)$.

In the absence of noise the system (10) has a periodic metastable state (attractor) $q_{a}(t)=q_{a}\left(t+\tau_{F}\right)$ and a periodic boundary $q_{b}(t)=q_{b}\left(t+\tau_{F}\right)$ of the basin of attraction to $q_{a}(t)$, see Fig. I(b). The states $q_{a}(t)$ and $q_{b}(t)$ are, respectively, the stable and unstable periodic solutions of the equation $\dot{q}=K(q, t)$. For concreteness we assume that $q_{a}(t)<q_{b}(t)$.

The PPD $p_{h}\left(q, t \mid q_{f}, t_{f}\right)$ as defined in the Introduction is the conditional probability density (with respect to $q$ ) of passing through a point $(q, t)$ in the coordinate-time space on the way from the attractor to a point $\left(q_{f}, t_{f}\right)$. It has the form [1]

$$
p_{h}\left(q, t \mid q_{f}, t_{f}\right)=\frac{\rho\left(q_{f}, t_{f} \mid q, t\right) \rho\left(q, t \mid q_{\mathrm{in}}, t_{\mathrm{in}}\right)}{\rho\left(q_{f}, t_{f} \mid q_{\mathrm{in}}, t_{\mathrm{in}}\right)},
$$

where $\rho\left(q_{1}, t_{1} \mid q_{2}, t_{2}\right)$ is the probability density (with respect to $\left.q_{1}\right)$ of a transition from $\left(q_{2}, t_{2}\right)$ to $\left(q_{1}, t_{1}\right)$, with $t_{1}>t_{2}$.

We assume that the noise intensity $D$ is small. Then the period-average escape rate is $\bar{W} \propto \exp (-R / D)$, where $R$ is the activation energy of escape, $R \gg D$ 16, 17, 18, 19, 20]. The condition on $D$ is that $\bar{W}^{-1}$ largely exceeds the relaxation time of the system $t_{\mathrm{r}}$ and the modulation period $\tau_{F}$. Eq. (2) gives the distribution of escape paths in a broad time range

$$
\bar{W}^{-1} \gg t_{f}-t_{\text {in }}>t-t_{\text {in }} \gg t_{\mathrm{r}}, \tau_{F},
$$

where the population of the attraction basin practically does not change. For $t-t_{\text {in }} \gg t_{\mathrm{r}}$ the initial state $q_{\text {in }}$, which is close to the attractor $q_{a}\left(t_{\text {in }}\right)$, gets forgotten. 
Then the right-hand side of Eq. (2) becomes independent of $t_{\text {in }}$.

The functions $\rho\left(q, t \mid q_{\text {in }}, t_{\text {in }}\right)=\rho(q, t)$ and $\rho\left(q_{f}, t_{f} \mid q_{\text {in }}, t_{\text {in }}\right)=\rho\left(q_{f}, t_{f}\right)$ give the time-periodic probability density to find the system in states $q$ and $q_{f}$, respectively. To study escape pathways we calculate the PPD for $q_{f}$ outside the attraction basin, $q_{f}>q_{b}\left(t_{f}\right)$.

The transition probability density $\rho\left(q, t \mid q^{\prime}, t^{\prime}\right)$ is a solution of the Fokker-Planck equation (FPE)

$$
\partial_{t} \rho=-\partial_{q}[K(q, t) \rho]+D \partial_{q}^{2} \rho,
$$

with the initial condition $\rho\left(q, t^{\prime} \mid q^{\prime}, t^{\prime}\right)=\delta\left(q-q^{\prime}\right)$. Even for a $1 \mathrm{D}$ system, this equation does not have a known explicit solution except for the case of $K$ linear in $q$. To analyze the PPD we will have to find approximate solutions in different regions and match them.

In the following subsections we discuss the dynamics of the system prior and during escape.

\section{A. Dynamics near the periodic states}

We start with the dynamics close to the periodic states $q_{i}(t), i=a, b$. In the absence of noise it is described by the linear equations $\delta \dot{q}=\mu_{i}(t) \delta q$ with $\delta q=q-q_{i}(t)$ and

$$
\mu_{i}(t)=\mu_{i}\left(t+\tau_{F}\right)=\left[\partial_{q} K\right]_{q_{i}(t)}, \quad i=a, b .
$$

Time evolution of the deviation $\delta q$ from $q_{i}(t)$ is given by

$$
\begin{aligned}
& \delta q(t)=\delta q\left(t^{\prime}\right) \kappa_{i}\left(t, t^{\prime}\right), \\
& \kappa_{i}\left(t, t^{\prime}\right)=\exp \left[\int_{t^{\prime}}^{t} d \tau \mu_{i}(\tau)\right], \quad i=a, b .
\end{aligned}
$$

Because of the periodicity of $\mu_{i}(t)$, the Floquet multipliers

$$
M_{i}=\kappa_{i}\left(t+\tau_{F}, t\right)=\exp \left(\bar{\mu}_{i} \tau_{F}\right), \quad i=a, b,
$$

are independent of time, with $M_{a}<1$ and $M_{b}>1$. In Eq. (7) $\bar{\mu}_{i}(i=a, b)$ is the period-average value of $\mu_{i}(t)$, with $\bar{\mu}_{a}<0, \bar{\mu}_{b}>0$. The relaxation time of the system can be chosen as $t_{\mathrm{r}}=\bar{\mu}_{b}^{-1} \sim\left|\bar{\mu}_{a}\right|^{-1}$.

Weak noise leads to fluctuations about the attractor, which have Gaussian distribution near the maximum in the time range (3). From Eq. (4) this distribution has the form

$$
\rho_{a}(q, t)=G\left(q-q_{a}(t) ; \sigma_{a}(t)\right)
$$

with

$$
G(x ; \sigma)=\left(2 \pi D \sigma^{2}\right)^{-1 / 2} \exp \left(-x^{2} / 2 D \sigma^{2}\right) .
$$

The variance $\sigma_{a}^{2}(t)$ is periodic in time. It is given by the expression

$$
\sigma_{i}^{2}(t)=2\left|M_{i}^{-2}-1\right|^{-1} \int_{0}^{\tau_{F}} d t_{1} \kappa_{i}^{-2}\left(t+t_{1}, t\right)
$$

with $i=a$.

\section{B. Most probable escape paths}

Along with small fluctuations, there also happen occasional large deviations from $q_{a}(t)$, including escape from the attraction basin. In a broad parameter range escape events are very strongly synchronized with the modulation, see Ref. 20 and papers cited therein. Because large fluctuations have small probabilities, in escape the system closely follows the trajectory that is least improbable among all possible escape trajectories. As mentioned in the Introduction, it is usually called the most probable escape path, $q_{\text {opt }}(t)$. Inside the attraction basin, escape trajectories lie within periodically repeated narrow tubes. The tubes have a width $\sim l_{D}$ and are centered at the MPEP's $q_{\text {opt }}^{(n)}(t) \equiv q_{\text {opt }}\left(t+n \tau_{F}\right), n=0, \pm 1, \ldots$.

The MPEP's provide a solution to the variational problem of maximizing the probability of a fluctuation in which the system moves from $q_{a}(t)$ to $q_{b}(t)$. This problem can be mapped onto the problem of dynamics of an auxiliary Hamiltonian system. The latter is described by the Wentzell-Freidlin Hamiltonian $H(p, q ; t)=p^{2}+p K(q, t)$ 22]. Its equations of motion have the form

$$
\dot{q}=K(q, t)+2 p, \quad \dot{p}=-p \partial_{q} K .
$$

The MPEP's correspond to the minimal action heteroclinic Hamiltonian trajectories $\left(q_{\text {opt }}(t), p_{\text {opt }}(t)\right)$ [16, 23]. They start for $t \rightarrow-\infty$ at the periodic hyperbolic state $\left(q_{a}(t), p=0\right)$ of the auxiliary system and for $t \rightarrow \infty$ approach its another periodic hyperbolic state, $\left(q_{b}(t), p=\right.$ $0)$.

Well outside the diffusion regions around the periodic states $q_{a, b}(t)$ the motion along the MPEP is fast. It is seen from Eq. (11) that the system moves between these regions over time $t_{\mathrm{r}}$. Close to $q_{b}(t)$ the system is slowed down, and in the region $\left|q-q_{b}(t)\right| \lesssim l_{D}$ the motion is dominated by diffusion. The duration of staying in the vicinity of $q_{b}(t)$ can be obtained by linearizing the equation of motion (11) near $q_{b}$ and is given by the Suzuki time $t_{S} \sim \bar{\mu}_{b}^{-1} \ln \left(\Delta q / l_{D}\right)$ [24, 25], where $\Delta q=\min _{t}\left|q_{b}(t)-q_{a}(t)\right|$ is the typical distance between the periodic states.

Periodically modulated systems are advantageous as they allow one to observe, via the prehistory distribution, both the fast motion along the MPEP and the slow motion near the unstable state. As we show below, the peak of the PPD does not display broadening due to diffusion near $q_{b}$, as does the PPD in the absence of modulation $15]$.

\section{PREHISTORY PROBABILITY DISTRIBUTION NEAR THE BASIN BOUNDARY}

We will calculate the PPD in the regime of strong synchronization of escape 19, 20]. In this regime the probability distribution $\rho(q, t)$ of finding a particle behind the basin boundary has the form of sharp periodic pulses as 
a function of $t$. It is most interesting to find the PPD for a final point $\left(q_{f}, t_{f}\right)$ on the $(q, t)$ plane near the center of such a pulse.

We will assume that the point $\left(q_{f}, t_{f}\right)$ is sufficiently far from the diffusion-dominated layer (region B in Fig. 1) around the basin boundary, so that the distance to the boundary is $Q_{f}=q_{f}-q_{b}\left(t_{f}\right) \gg l_{D}$. This condition is usually realized in experiments, where the position of a particle detector is chosen so as to ensure that the detected particles have practically no chance to return to the attraction basin. At the same time we assume for convenience that the final point is still in the harmonic region, $Q_{f} \ll \Delta q$, in which case the motion between $q_{b}$ and $q_{f}$ can be described by linearizing equation (1) in $Q=q-q_{b}(t)$.

We will start the analysis of the $\mathrm{PPD} p_{h}\left(q, t \mid q_{f}, t_{f}\right)$ with the case where not only $q_{f}$ but also the point $(q, t)$ through which the trajectory of interest has passed is also in the harmonic region around the basin boundary $q_{b}(t)$.

\section{A. Transition probability density}

As seen from Eq. (2), finding the PPD requires calculating the transition probability density $\rho\left(q_{f}, t_{f} \mid q, t\right)$. The Fokker-Planck equation for $\rho\left(q_{f}, t_{f} \mid q, t\right)$ can be linearized near $q_{b}(t)$,

$$
\partial_{t_{f}} \rho=-\mu_{b}\left(t_{f}\right) \partial_{Q_{f}}\left(Q_{f} \rho\right)+D \partial_{Q_{f}}^{2} \rho,
$$

where $\rho=\rho\left(q_{f}, t_{f} \mid q, t\right)$ and $Q_{f}=q_{f}-q_{b}\left(t_{f}\right)$.

The solution of Eq. (12) can be sought in the form of a Gaussian distribution

$$
\rho=G\left(Q_{f}-\tilde{Q}\left(t_{f}\right) ; \tilde{\sigma}\left(t_{f}\right)\right),
$$

with $G(x ; \sigma)$ given by Eq. (9). Eq. (12) will be satisfied provided the hitherto unknown functions $\tilde{\sigma}, \tilde{Q}$ obey the equations

$$
\frac{d \tilde{\sigma}^{2}}{d t_{f}}=2 \mu_{b}\left(t_{f}\right) \tilde{\sigma}^{2}+2, \quad \frac{d \tilde{Q}}{d t_{f}}=\mu_{b}\left(t_{f}\right) \tilde{Q} .
$$

The initial conditions for these equations follow from the condition $\rho\left(q_{f}, t \mid q, t\right)=\delta\left(q_{f}-q\right)$. They have the form $\tilde{\sigma}^{2}(t)=0$ and $\tilde{Q}(t)=Q=q-q_{b}(t)$. Then the solution of Eqs. 114 is

$$
\begin{aligned}
& \tilde{\sigma}^{2}\left(t_{f}\right)=2 \int_{t}^{t_{f}} d \tau \kappa_{b}^{2}\left(t_{f}, \tau\right) \\
& \tilde{Q}\left(t_{f}\right)=Q \kappa_{b}\left(t_{f}, t\right)
\end{aligned}
$$

Finally, using the function

$$
\sigma_{f}^{2}\left(t_{f}, t\right)=\kappa_{b}^{-2}\left(t_{f}, t\right) \tilde{\sigma}^{2}\left(t_{f}\right)=2 \int_{t}^{t_{f}} d \tau \kappa_{b}^{-2}(\tau, t)
$$

we can write the distribution in the form

$$
\rho=\kappa_{b}^{-1}\left(t_{f}, t\right) G\left(Q-Q_{f} \kappa_{b}^{-1}\left(t_{f}, t\right) ; \sigma_{f}\left(t_{f}, t\right)\right) .
$$

The function $Q_{f} \kappa_{b}^{-1}\left(t_{f}, t\right)$ has a simple meaning. Consider the noise-free trajectory that passes through the point $Q_{f}$ at time $t_{f}$. This trajectory should have passed through the point $Q_{f} \kappa_{b}^{-1}\left(t_{f}, t\right)$ at time $t$. As expected, the transition probability (17) is maximal for $Q$ coinciding with this point.

The function $\sigma_{f}^{2}$, Eq. (16), is simply related to the function $\sigma_{b}^{2}(t), \mathrm{Eq}$. (10), introduced earlier,

$$
\sigma_{b}^{2}(t)-\kappa_{b}^{-2}\left(t_{f}, t\right) \sigma_{b}^{2}\left(t_{f}\right)=\sigma_{f}^{2}\left(t_{f}, t\right) .
$$

We will use this relation in what follows.

\section{B. General expression for the PPD near $q_{b}(t)$}

From Eq. (2), the PPD is determined by the product of the transition probability (17) and the ratio of the quasiperiodic distributions $\rho(q, t) / \rho\left(q_{f}, t_{f}\right)$. The distribution $\rho(q, t)$ close to $q_{b}(t)$ was found earlier [19, 20]. It has the form

$$
\begin{aligned}
& \rho(q, t)=\int_{0}^{\infty} d p \rho^{\prime}(p, Q, t), \quad Q=q-q_{b}(t) \\
& \rho^{\prime}(p, Q, t)=\frac{\mathcal{E}}{\sqrt{D}} \exp \left\{-\frac{1}{D}\left[\frac{p^{2} \sigma_{b}^{2}(t)}{2}+p Q+s(\phi)\right]\right\} \\
& \phi(p, t)=\Omega_{F} \ln \left[\frac{p \kappa_{b}\left(t, t^{\prime}\right)}{\bar{\mu}_{b} l_{D}}\right], \quad \Omega_{F}=\omega_{F} / \bar{\mu}_{b} .
\end{aligned}
$$

Here $\mathcal{E}$ and $t^{\prime}$ are constants, $s(\phi)=s(\phi+2 \pi)$ is a zeromean $2 \pi$-periodic function, and $\Omega_{F}$ is the dimensionless modulation frequency.

The function $s(\phi)$ in Eq. (19) plays the role of an instantaneous modulation-induced change of the activation energy. In the regime of strong synchronization of escape the minimal value $s_{\mathrm{m}}$ of $s(\phi)$ satisfies the condition $\left|s_{\mathrm{m}}\right| \gg D$. The minima of $s(\phi)$ lie on the optimal escape paths [19, 20], $p=p_{\text {opt }}^{(n)}(t) \equiv p_{\text {opt }}\left(t+n \tau_{F}\right)$. Here $n=0, \pm 1, \ldots$ enumerates periodically repeated MPEP's, see Fig. 1(b); we set $\left(q_{\mathrm{opt}}^{(0)}(t), p_{\mathrm{opt}}^{(0)}(t)\right)=\left(q_{\mathrm{opt}}(t), p_{\mathrm{opt}}(t)\right)$.

Near the basin boundary $q_{b}(t)$ the optimal paths satisfy the linearized equations (11) and evolve in time as

$$
\begin{aligned}
& p_{\mathrm{opt}}(t)=\kappa_{b}^{-1}\left(t, t^{\prime}\right) p_{\mathrm{opt}}\left(t^{\prime}\right), \quad Q_{\mathrm{opt}}(t)=-\sigma_{b}^{2}(t) p_{\mathrm{opt}}(t), \\
& Q_{\mathrm{opt}}(t)=q_{\mathrm{opt}}(t)-q_{b}(t), \quad\left|Q_{\mathrm{opt}}\right| \ll \Delta q .
\end{aligned}
$$

Eq. (20) describes how a given optimal path approaches $\left(q_{b}(t), p=0\right)$ for $t \rightarrow \infty$. The parameter $t^{\prime}$ is determined by matching to one of the periodically repeated trajectories (11) that start from $\left(q_{a}(t), p=0\right)$ for $t \rightarrow-\infty$.

Expanding the function $s(\phi)$ around its minima at the periodically repeated MPEP's, to second order in $\phi(p, t)-\phi\left(p_{\mathrm{opt}}^{(n)}(t), t\right)$ we obtain the probability distribution (19) as a sum of contributions from the MPEP's,

$$
\begin{aligned}
& \rho^{\prime}(p, Q, t)=\frac{\mathcal{E} e^{-s_{\mathrm{m}} / D}}{\sqrt{D}} \sum_{n} \exp \left[-r^{(n)}(p, Q, t) / D\right] \\
& r^{(n)}=p Q+\frac{p^{2} \sigma_{b}^{2}(t)}{2}+\frac{\Omega_{F}^{2} s_{\mathrm{m}}^{\prime \prime}}{2} \ln ^{2}\left[\frac{p}{p_{\mathrm{opt}}^{(n)}(t)}\right]
\end{aligned}
$$


Here $s_{\mathrm{m}}^{\prime \prime} \equiv\left(d^{2} s / d \phi^{2}\right)_{\mathrm{m}}$ is the curvature of the function $s$ at the minimum. For strong synchronization, where $\left|s_{\mathrm{m}}\right| \gg D$, we have $s_{\mathrm{m}}^{\prime \prime} \gg D$ as well, which is a consequence of $s(\phi)$ being a zero-mean periodic function. The quantity $s_{\mathrm{m}}^{\prime \prime}$ can be found [19, 20], along with the constant $\mathcal{E} \exp \left(-s_{\mathrm{m}} / D\right)$, by matching the periodic distribution (19) to the distribution well inside the attraction basin.

It follows from Eq. (20) that near the basin boundary

$$
p_{\mathrm{opt}}^{(n+k)}(t)=p_{\mathrm{opt}}^{(n)}\left(t+k \tau_{F}\right)=M_{b}^{-k} p_{\mathrm{opt}}^{(n)}(t) .
$$

With account taken of the relation $M_{b}=\exp \left(2 \pi / \Omega_{F}\right)$, this leads to the expression

$$
r^{(n+k)}=r^{(n)}+2 \pi k \Omega_{F} s_{\mathrm{m}}^{\prime \prime} \ln \left[\frac{p}{p_{\mathrm{opt}}^{(n)}(t)}\right]+2 \pi^{2} k^{2} s_{\mathrm{m}}^{\prime \prime} .
$$

Combining Eqs. (17) and (21) and using Eq. (18) we obtain for the PPD near the basin boundary,

$$
\begin{aligned}
& p_{h}\left(q, t \mid q_{f}, t_{f}\right) \\
& =\kappa_{b}^{-1}\left(t_{f}, t\right) G\left(Q-Q_{f} \kappa_{b}^{-1}\left(t_{f}, t\right) ; \sigma_{f}\left(t_{f}, t\right)\right) \\
& \times \frac{\sum_{n} \int_{0}^{\infty} d p \exp \left[-r^{(n)}(p, Q, t) / D\right]}{\sum_{n} \int_{0}^{\infty} d p \exp \left[-r^{(n)}\left(p, Q_{f}, t_{f}\right) / D\right]} .
\end{aligned}
$$

This expression gives the general form of the PPD near the basin boundary. We note that, even though the equations of motion near $q_{b}(t)$ can be linearized, the PPD is generally non-Gaussian. This distortion is an important feature of escape dynamics.

\section{ADIABATIC REGIME NEAR THE BASIN BOUNDARY}

We start the analysis with the case of slow modulation, $\Omega_{F} \ll 1$, where the motion can be described in the adiabatic approximation. As we show below, in the adiabatic regime and for strong synchronization, $s_{\mathrm{m}}^{\prime \prime} \gg D$, only one term contributes to each of the sums in Eq. (24). The shape of the PPD in this case is determined by the parameter

$$
\theta=\Omega_{F}^{2} s_{\mathrm{m}}^{\prime \prime} / D .
$$

We call $\theta$ the distortion parameter. This is because for $\theta \ll 1$ the escape current has a form of Gaussian peaks, whereas for larger $\theta$ the peaks of the current become nonGaussian [19, 20]. Formally, $\theta$ is equal to the ratio of two small parameters, the squared reduced modulation frequency $\omega_{F} / \bar{\mu}_{b}=\Omega_{F}$ and the noise intensity scaled by the effective modulation strength $D / s_{\mathrm{m}}^{\prime \prime}$.

The physical meaning of $\theta$ can be understood in the following way. In the adiabatic picture one usually thinks of escape as occurring in the instantaneous potential $U(q, t)$. Most likely it happens once per period at the time $t_{\mathrm{m}}$ when the barrier height $\Delta U(t)=U\left(q_{b}(t), t\right)-U\left(q_{a}(t), t\right)$ is minimal, cf. Fig. 1 As explained in the Introduction, the typical width of the time window for escape $\Delta t$ is determined by the condition $\left[d^{2} \Delta U / d t^{2}\right]_{\mathrm{m}}(\Delta t)^{2}=D$, where the subscript $m$ indicates that the derivative is evaluated for $t=t_{\mathrm{m}}$. The parameter $s_{\mathrm{m}}^{\prime \prime}$ is (see Ref 20)

$$
s_{\mathrm{m}}^{\prime \prime}=\left[d^{2} \Delta U / d t^{2}\right]_{\mathrm{m}} / \Omega_{F}^{2} \mu_{b}\left(t_{\mathrm{m}}\right) \sim\left[d^{2} \Delta U / d t^{2}\right]_{\mathrm{m}} / \omega_{F}^{2} .
$$

Therefore the parameter $\theta \sim\left[(\Delta t)^{2} \bar{\mu}_{b}^{2}\right]^{-1}$ is the squared ratio of the relaxation time of the system $t_{\mathrm{r}}=\bar{\mu}_{b}^{-1}$ to $\Delta t$. It shows whether the system moves fast enough to escape while the barrier remains at its minimum or the barrier noticeably changes during escape leading to a delay of escape with respect to $t_{\mathrm{m}}$ and a distortion of the tube of escape trajectories.

Because the PPD evolves over time $\sim t_{\mathrm{r}}$, of interest is the time range $\left|t-t_{f}\right| \lesssim t_{\mathrm{r}} \ll \tau_{F}$. Moreover, both $t$ and $t_{f}$ should be close to $t_{\mathrm{m}}$. Then the instantaneous relaxation rate $\mu_{b}(t)$ can be approximated by its value $\mu_{b \mathrm{~m}} \equiv \mu_{b}\left(t_{\mathrm{m}}\right)$. The functions $\kappa_{b}$ and $\sigma_{f}^{2}$ become

$$
\begin{aligned}
\kappa_{b \mathrm{~m}}\left(t_{f}, t\right) & =\exp \left[\mu_{b \mathrm{~m}}\left(t_{f}-t\right)\right], \\
\sigma_{f \mathrm{~m}}^{2}\left(t_{f}, t\right) & =\sigma_{b \mathrm{~m}}^{2}\left\{1-\exp \left[-2 \mu_{b \mathrm{~m}}\left(t_{f}-t\right)\right]\right\},
\end{aligned}
$$

where

$$
\sigma_{b}^{2}(t) \approx \sigma_{b}^{2}\left(t_{\mathrm{m}}\right) \equiv \sigma_{b \mathrm{~m}}^{2}=1 / \mu_{b \mathrm{~m}} .
$$

\section{A. Weak distortion, $\theta \ll 1$}

We consider first the limit of weak distortion, $\theta \ll 1$ (more precisely, the weak distortion condition has the form $\theta \ln ^{2}\left(s_{\mathrm{m}}^{\prime \prime} / D\right) \ll 1$, see below). In this limit one can think of motion in the fully adiabatic way, assuming that it occurs in a quasistatic potential $U\left(q, t_{\mathrm{m}}\right)$. The periodic distribution behind the attraction basin $\rho\left(q_{f}, t\right)$, which is proportional to the instantaneous escape rate, has a form of periodic in time Gaussian pulses, with width $\sim$ $\left(D / s_{\mathrm{m}}^{\prime \prime}\right)^{1 / 2} \tau_{F}[20]$.

For small $\theta$ expression (24) for the PPD can be simplified. We will start with the analysis of the denominator in this expression. If there were no term $\propto \Omega_{F}^{2} s_{\mathrm{m}}^{\prime \prime} / D=\theta$ in $r^{(n)}\left(p, Q_{f}, t_{f}\right) / D$, the typical values of $p$ contributing to the integral over $p$ would be $\sim D / Q_{f}$. For such $p$ there may be only one $n$ for which the term $\theta \ln ^{2}\left[p / p_{\text {opt }}^{(n)}\left(t_{f}\right)\right]$ in $r^{(n)} / D$ is small, whereas for all other $n$ it is $\propto s_{\mathrm{m}}^{\prime \prime} / D$ [cf. Eq. [23)], making the integrand exponentially small. A similar argument applies to the numerator in Eq. (24), except that, depending on $Q$, the typical values of $p$ are of order of $\left(D / \mu_{b \mathrm{~m}}\right)^{1 / 2}, D / Q$, or $-\mu_{b \mathrm{~m}} Q$.

Keeping only the leading term in the sums in the numerator and denominator of Eq. (24) and disregarding corrections $\propto \theta$, we obtain

$$
\begin{aligned}
& p_{h}\left(q, t \mid q_{f}, t_{f}\right)=G\left(Q-Q_{f} \kappa_{b \mathrm{~m}}^{-1}\left(t_{f}, t\right) ; \sigma_{f \mathrm{~m}}\left(t_{f}, t\right)\right) \\
& \times \frac{Q_{f} \kappa_{b \mathrm{~m}}^{-1}\left(t_{f}, t\right)}{\sqrt{2 D \sigma_{b \mathrm{~m}}^{2}}} \exp \left[\frac{Q^{2}}{2 D \sigma_{b \mathrm{~m}}^{2}}\right] \operatorname{erfc}\left[\frac{Q}{\sqrt{2 D \sigma_{b \mathrm{~m}}^{2}}}\right] .
\end{aligned}
$$


In deriving this expression we also took into account that the final point $q_{f}$ is sufficiently far from the diffusiondominated region behind the basin boundary, $Q_{f} \gg l_{D}$, and disregarded corrections $\sim l_{D}^{2} / Q_{f}^{2}$. We note that the terms $\propto \theta$ in $r^{(n)}$ in the numerator and denominator in Eq. (24) have a logarithmic factor. This factor may become large in the weak-noise limit. One can show that for the corrections $\propto \theta$ to be small it is necessary that $\theta \ln ^{2}\left(s_{\mathrm{m}}^{\prime \prime} / D\right) \ll 1$. This condition is equivalent to the inequality $\Delta t \gg t_{S}$, where $\Delta t$ is the characteristic time window within which the barrier height is practically constant and $t_{S}$ is the Suzuki time, see Eq. (28) below.

Equation (27) for the PPD is further simplified for such times that the point $Q_{f} \kappa_{b}^{-1}\left(t_{f}, t\right)$ is far behind the basin boundary compared to $l_{D}$. This is the point that the noise-free trajectory arriving at $Q_{f}$ at time $t_{f}$ passes at time $t$. For such $t$, the PPD as a function of $Q$ has the form of a Gaussian peak with variance $D \sigma_{f}^{2}\left(t_{f}, t\right)$. This means that the trajectories arriving to the point $Q_{f}$ are close to the noise-free trajectory. The tube of these trajectories is diffusion-broadened, with width $\propto$ $\left[D\left(t_{f}-t\right)\right]^{1 / 2}$ for small $t_{f}-t$.

Because for $\theta \ll 1$ escape as a whole occurs in the quasistatic potential $U\left(q, t_{\mathrm{m}}\right)$, the full calculation of the PPD described in Section VI leads to the same result as in the case of escape in a stationary potential studied earlier [15]. With increasing $t_{f}-t$ the peak of the PPD (27) crosses the basin boundary and enters the attraction basin. This is the slowest part of the PPD evolution. Its duration is determined by the Suzuki time

$$
t_{S}=t_{\mathrm{r}} \ln \left(s_{\mathrm{m}}^{\prime \prime} / D\right) .
$$

The peak is sharply broadened in this region. Deep on the intrawell side, $-Q \gg l_{D}$, the width of the peak becomes independent of $D$, i.e. parametrically larger than the diffusion-limited width $\sim l_{D}$. As $t_{f}-t$ increases further, the PPD peak approaches the attractor and narrows down, with the width becoming again diffusion-limited. This part of the evolution takes $\sim t_{\mathrm{r}}$.

\section{B. Strong distortion, $\theta \gg 1$}

The shape of the PPD near the maximum changes dramatically in the range where the modulation is still dynamically slow, $\Omega_{F} \ll 1$, but the distortion parameter $\theta$ becomes large, $\theta \gg 1$. Here, the shape of the potential barrier for escape changes as the particle crosses the diffusion region around the basin boundary. This leads to a strong change of the PPD compared to the picture based on the quasistatic barrier that was discussed before.

For $\theta \gg 1$ in an important range of $Q, t$ and $Q_{f}, t_{f}$ the integrands in the numerator and denominator in Eq. (24) have sharp extrema as functions of $p$ for $p=p_{\text {opt }}^{(n)}$ (a more precise condition is specified below). Integration over $p$ can be done by the steepest descent method. It gives the PPD in the form of a Gaussian distribution over $Q$ with time-dependent center $Q_{n_{0}}$ and variance $D \sigma_{f}^{2}$,

$$
p_{h}\left(q, t \mid q_{f}, t_{f}\right)=G\left(Q-Q_{n_{0}} ; \sigma_{f}\left(t_{f}, t\right)\right)
$$

where

$$
\begin{aligned}
Q_{n} & \equiv Q_{n}\left(t \mid q_{f}, t_{f}\right) \\
& =Q_{\mathrm{opt}}^{(n)}(t)+\left(Q_{f}-Q_{\mathrm{opt}}^{(n)}\left(t_{f}\right)\right) \kappa_{b}^{-1}\left(t_{f}, t\right)
\end{aligned}
$$

and $n_{0} \equiv n_{0}\left(q_{f}, t_{f}\right)$. Here we have taken into account that the major contribution to each of the sums in Eq. (24) comes from one term, with $n=n_{0}$.

It is clear from the analysis of the denominator in Eq. (24) that the value of $n_{0}$ is determined by the condition that $p_{\mathrm{opt}}^{\left(n_{0}\right)}\left(t_{f}\right) Q_{f} / D \sim 1$ for the $n_{0}$ th optimal path. The very existence of such $n_{0}$ follows from the fact that we consider $t_{f}$ for which the probability density $\rho\left(q_{f}, t_{f}\right)$ is close to its maximum over $t_{f}$; this maximum is reached once per period for $p_{\mathrm{opt}}^{(n)}\left(t_{f}\right) Q_{f} / D=1$ [19, 20]. The terms with $\left|n-n_{0}\right| \geq 1$ in the denominator in Eq. (24) are

$$
\propto M_{b}^{n_{0}-n} \exp \left(-M_{b}^{n_{0}-n}\right) \ll 1
$$

where we have used $M_{b}=\exp \left(2 \pi / \Omega_{F}\right) \gg 1$ (this estimate is written for $p_{\mathrm{opt}}^{\left(n_{0}\right)}\left(t_{f}\right) Q_{f} / D=1$ ).

A simple qualitative argument shows that the same $n_{0}$ gives a major contribution to the sum in the numerator of Eq. (24). Indeed, the terms with different $n$ correspond to $t$ changing by an integer number of the modulation periods $\tau_{F}$, whereas in its central part (section $\mathrm{C}$ in Fig. 1) an optimal escape trajectory lasts for the time small compared to $\tau_{F}$. Therefore $\left|t-t_{f}\right| \ll \tau_{F}$. The formal condition for this approximation to be true is that the Suzuki time $t_{S}$ (28) is small compared to the modulation period.

The maximum of the Gaussian PPD peak (29) lies at $Q_{n_{0}}\left(t \mid q_{f}, t_{f}\right)$. This function is a sum of the optimal path $Q_{\mathrm{opt}}^{(n)}$, which is located inside the attraction basin, and the decaying in time term $\propto Q_{f}-Q_{\text {opt }}^{(n)}\left(t_{f}\right)$, which is determined by the final point $\left(q_{f}, t_{f}\right)$ and is located outside the attraction basin. Its time dependence is particularly simple in the case where $t_{f}$ corresponds to the maximum of the distribution $\rho\left(q_{f}, t_{f}\right)$, i.e., $p_{\mathrm{opt}}^{\left(n_{0}\right)}\left(t_{f}\right)=D / Q_{f}$,

$$
Q_{n_{0}}\left(t \mid Q_{f}, t_{f}\right) \approx Q_{f} e^{-\mu_{b \mathrm{~m}}\left(t_{f}-t\right)}-\frac{D}{\mu_{b \mathrm{~m}} Q_{f}} e^{\mu_{b \mathrm{~m}}\left(t_{f}-t\right)}
$$

The first term in $Q_{n_{0}}$, Eq. (31), decreases with increasing $t_{f}-t$, which describes approaching the basin boundary backward in time. The second term, on the other hand, increases with $t_{f}-t$; this term describes the motion, backward in time, from the boundary to the interior of the attraction basin. The motion at the boundary is initiated by noise, and therefore this term is $\propto D$. For sufficiently long $t_{f}-t$ the distribution maximum $Q_{n_{0}}$ approaches the optimal path $Q_{\mathrm{opt}}^{\left(n_{0}\right)}(t)$. The overall behavior 

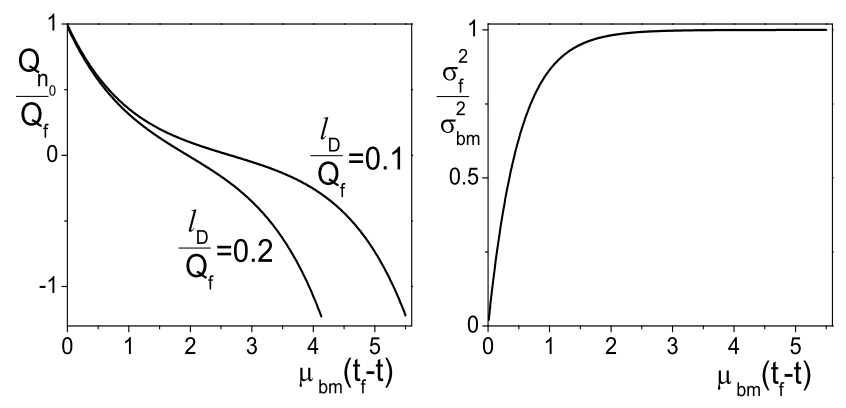

FIG. 3: (Color online) The reduced position of the maximum (left) and the reduced width (right) of the PPD (29) in the adiabatic regime as a function of the reduced time $\mu_{b \mathrm{~m}}\left(t_{f}-t\right)$.

of $Q_{n_{0}}$ is shown in Fig. 3 It is seen that the motion is slowed down near the basin boundary, and the slowing down strongly depends on the noise intensity, in agreement with Eq. (31).

The width of the Gaussian PPD peak (29) is diffusionlimited; its time dependence is given by Eq. (26) and is also simple. It is shown in the right panel of Fig. [3.

The condition that the integrands over $p$ in Eq. (24) are maximal for $p_{\text {opt }}^{\left(n_{0}\right)}$ imposes a limitation on $Q$ where the explicit expression for the PPD (29) applies. For $Q$ close to the maximum of the distribution, $\left|Q-Q_{n_{0}}\right| \lesssim l_{D}$, this condition takes the form $\theta \gg\left|Q_{\mathrm{opt}}^{\left(n_{0}\right)}(t) / l_{D}\right|$. Therefore expression (29) describes the distribution not only in the whole range between $\left(Q_{f}, t_{f}\right)$ and the basin boundary, but also throughout the diffusion region around the basin boundary and a region that goes deeper into the attraction basin.

\section{NONADIABATIC REGIME NEAR THE BASIN BOUNDARY}

The PPD can display a qualitatively different behavior for nonadiabatic modulation, $\Omega_{F} \sim 1$. With increasing $t_{f}-t$ the PPD inside the attraction basin can split into several peaks. Such splitting occurs already close to the basin boundary and is described by the general expression (24). Formally, the PPD has well-resolved multiple peaks when several terms in the numerator of Eq. (24) are of the same order of magnitude.

In the nonadiabatic regime necessarily $\theta \gg 1$. Therefore integration over $p$ in Eq. (24) can be done by the steepest descent method. The integrands are maximal for $p=p_{\mathrm{opt}}^{(n)}$. Using Eq. (18) one can show that each term in the numerator gives a Gaussian distribution over $Q$ centered at $Q_{n} \equiv Q_{n}\left(t \mid q_{f}, t_{f}\right)$, Eq. (30), with variance $D \sigma_{f}^{2}\left(t_{f}, t\right)$, Eq. (16). This is similar to the adiabatic case (29), except that now the PPD is a sum of appropriately weighted Gaussian peaks,

$$
p_{h}\left(q, t \mid q_{f}, t_{f}\right)=\frac{\sum_{n} B_{n} G\left(Q-Q_{n} ; \sigma_{f}\left(t_{f}, t\right)\right)}{\sum_{n} B_{n}} .
$$

The weighting factors $B_{n}$ are

$$
\begin{aligned}
& B_{n} \equiv B_{n}\left(q_{f}, t_{f}\right)=x_{n} \exp \left(-x_{n}\right), \\
& x_{n}=x_{n}\left(q_{f}, t_{f}\right)=p_{\mathrm{opt}}^{(n)}\left(t_{f}\right) Q_{f} / D .
\end{aligned}
$$

If we keep only one term in the numerator and denominator in Eq. (32), with the same $n=n_{0}$ given by the condition that $B_{n}$ is maximal for $n=n_{0}$, Eq. (32) goes over into Eq. (29). However, in the nonadiabatic case there are regimes where the PPD as a function of $(Q, t)$ may display several peaks. Eq. (32) gives their shapes near the maxima.

The number of peaks of $p_{h}$ depends on $Q, t$, and $\Omega_{F}$, as well as the final point $\left(q_{f}, t_{f}\right)$. As before, we choose the final point so that the probability density behind the basin boundary $\rho\left(q_{f}, t_{f}\right)$ is close to its maximum over $t_{f}$, which occurs for $x_{n_{0}}\left(q_{f}, t_{f}\right) \sim 1$. The amplitudes of different peaks are

$$
B_{n_{0}+k}=x_{n_{0}} M_{b}^{-k} \exp \left(-x_{n_{0}} M_{b}^{-k}\right),
$$

with $M_{b}=\exp \left(2 \pi / \Omega_{F}\right)$ being the Floquet multiplier, Eq. (7). In practice, in the whole range of modulation parameters where escape is strongly synchronized the factor $M_{b}$ is comparatively large. Therefore for $x_{n_{0}} \sim 1$ the coefficients $B_{n_{0}+k}$ rapidly decay with increasing $|k|$, and only few peaks of the PPD can be simultaneously observed, primarily with non-negative $k=0,1, \ldots$.

The other condition for observing several peaks is that the distance between them exceed their width, that is in particular $\left|Q_{n_{0}}-Q_{n_{0}+1}\right| \gg l_{D}$. For $Q \approx Q_{n_{0}} \gg l_{D}$, i.e., when the PPD maximum is well behind the basin boundary with respect to the attraction basin, we have $\left|Q_{n_{0}}-Q_{n_{0}+1}\right| \sim\left|Q_{\mathrm{opt}}^{\left(n_{0}\right)}(t)\right| \sim l_{D}^{2} \kappa_{b}\left(t_{f}, t\right) / Q_{f} \ll l_{D}$, where we have used that $Q_{f} \gg l_{D}$ and that $\kappa_{b}\left(t_{f}, t\right) \sim 1$ when the PPD maximum is behind the boundary. Therefore in this region the PPD has indeed only one peak.

For longer $t_{f}-t$ when $\kappa_{b}\left(t_{f}, t\right) \gg 1$ and the peak positions $Q_{n_{0}}, Q_{n_{0}+1}$ are well inside the basin of attraction, the distance between them exceeds $l_{D}$. In this range the corresponding peaks of $p_{h}$ can be resolved. The ratio of their amplitudes is given by the factor $M_{b}$, as seen from Eq. (34). This is in good agreement with the results of numerical simulations for a specific model shown in Fig. 2 as will be discussed below.

We note that for $\Omega_{F} \gg 1$ synchronization of escape by modulation becomes weak. Although the system is far from the adiabatic limit, dynamics of the period-average coordinate is similar to dynamics in the case of a stationary system. Even though the factor $M_{b}$ becomes of order 1 , of interest is the PPD with respect to the periodaveraged coordinate, and this PPD does not display multiple peaks.

\section{THE PPD INSIDE THE ATTRACTION BASIN}

We assume throughout this Section that escape is strongly synchronized and $\theta \gg 1$. To find the PPD 
for $(q, t)$ deep inside the attraction basin we will use the equation

$$
p_{h}\left(q, t \mid q_{f}, t_{f}\right)=\int d q^{\prime} p_{h}\left(q, t \mid q^{\prime}, t^{\prime}\right) p_{h}\left(q^{\prime}, t^{\prime} \mid q_{f}, t_{f}\right) .
$$

It applies for $t<t^{\prime}<t_{f}$, as follows from the definition (2), and can be obtained [15] from the Chapman-Kolmogorov equation for the transition probability $\rho\left(q_{f}, t_{f} \mid q, t\right)$.

As we show, $t^{\prime}$ in Eq. (35) can be chosen in such a way that only a narrow range of $q^{\prime}$ contributes to the integral. The corresponding $q^{\prime}$ are close but not too close to $q_{b}\left(t^{\prime}\right)$. In this range both factors in the integrand, and hence the integral as a whole, can be explicitly calculated. In particular, the function $p_{h}\left(q^{\prime}, t^{\prime} \mid q_{f}, t_{f}\right)$ is given by Eq. (32).

The function $p_{h}\left(q, t \mid q^{\prime}, t^{\prime}\right)$ is the PPD inside the attraction basin. For a periodically modulated system it was found earlier [26]. Because in escape the system is likely to move close to one of the periodically repeated most probable escape paths $q_{\mathrm{opt}}^{(n)}(t)$, we are interested in $p_{h}\left(q, t \mid q^{\prime}, t^{\prime}\right)$ for both $(q, t)$ and $\left(q^{\prime}, t^{\prime}\right)$ lying close to such a path. If both $(q, t)$ and $\left(q^{\prime}, t^{\prime}\right)$ are close to an $n$th path $q_{\text {nnt }}^{(n)}(t)$, the corresponding PPD $p_{h}^{(n)}\left(q, t \mid q^{\prime}, t^{\prime}\right)$ is Gaussian [26],

$$
p_{h}^{(n)}\left(q, t \mid q^{\prime}, t^{\prime}\right)=G\left(q-q_{n}\left(t \mid q^{\prime}, t^{\prime}\right) ; \sigma_{n}\left(t^{\prime}, t\right)\right)
$$

Here $q_{n}\left(t \mid q^{\prime}, t^{\prime}\right)$ is the value of the coordinate at time $t$ on the optimal path that leads to the point $\left(q^{\prime}, t^{\prime}\right)$. This optimal path is described by Eq. (111).

The coordinate $q_{n}\left(t \mid q^{\prime}, t^{\prime}\right)$ as a function of time is close to $q_{\mathrm{opt}}^{(n)}(t)$. We can seek it in the form $q_{n}\left(t \mid q^{\prime}, t^{\prime}\right)=$ $q_{\text {opt }}^{(n)}(t)+\delta q_{n}\left(t \mid q^{\prime}, t^{\prime}\right)$. To the lowest order in the deviation of $\left(q^{\prime}, t^{\prime}\right)$ from $q_{\mathrm{opt}}^{(n)}\left(t^{\prime}\right)$, the function $\delta q_{n}$ can be found from linearized equations (11). This gives

$$
\begin{aligned}
& \delta \ddot{q}_{n}=V_{n}(t) \delta q_{n}, \\
& V_{n}(t)=\left[\partial_{q t} K+\frac{1}{2} \partial_{q q}\left(K^{2}\right)\right]_{q_{\mathrm{opt}}^{(n)}(t)} .
\end{aligned}
$$

The boundary conditions for $\delta q_{n}$ are $\delta q_{n}\left(t^{\prime} \mid q^{\prime}, t^{\prime}\right)=q^{\prime}-$ $q_{\text {opt }}^{(n)}\left(t^{\prime}\right)$ and $\lim _{t \rightarrow-\infty} \delta q_{n}\left(t \mid q^{\prime}, t^{\prime}\right)=0$; the latter condition simply means that the optimal path approaches the attractor $q_{a}(t)$ as $t \rightarrow-\infty$.

It follows from Eq. (37) that function $\beta_{n}(t)=\delta \dot{q}_{n} / \delta q_{n}$ satisfies a first-order (Riccati) equation,

$$
\dot{\beta}_{n}+\beta_{n}^{2}=V_{n}(t) .
$$

For $q^{\prime}, q_{\mathrm{opt}}^{(n)}\left(t^{\prime}\right)$ both close to the basin boundary $q_{b}\left(t^{\prime}\right)$, with account taken of the boundary conditions for $\delta q_{n}$ the solution of Eq. (37) can be written in terms of $\beta_{n}$ as

$$
\delta q_{n}\left(t \mid q^{\prime}, t^{\prime}\right)=\left(Q^{\prime}-Q_{\mathrm{opt}}^{(n)}\left(t^{\prime}\right)\right) \kappa_{n}\left(t, t^{\prime}\right)
$$

where

$$
\kappa_{n}\left(t, t^{\prime}\right)=\exp \left[\int_{t^{\prime}}^{t} d \tau \beta_{n}(\tau)\right] .
$$

We now discuss the asymptotic behavior of $\beta_{n}(t)$. For not too large $t^{\prime}-t$ the function $V_{n}(t)$ in Eqs. (37), (38) should be calculated for $q_{\mathrm{opt}}^{(n)}(t) \approx q_{b}(t)$. For such $V_{n}(t)$, the solution $\delta q_{n}(t)$ of Eq. (37) can either exponentially increase or decrease with increasing $t^{\prime}-t$. Of interest to us is the decreasing solution, which will ultimately go to zero for $t^{\prime}-t \rightarrow \infty$. A simple calculation shows that for this solution

$$
\beta_{n}(t) \approx \mu_{b}(t) \equiv\left[\partial_{q} K\right]_{q_{b}(t)}, \quad q_{\mathrm{opt}}^{(n)}(t) \approx q_{b}(t) .
$$

For much larger $t^{\prime}-t$ the optimal path $q_{\text {opt }}^{(n)}(t)$ approaches the attractor $q_{a}(t)$, and then the function $V_{n}(t)$ in Eqs. (37), (38) should be calculated for $q_{\mathrm{opt}}^{(n)}(t) \approx q_{a}(t)$. One can show that the solution $\delta q_{n}(t) \rightarrow 0$ for $t \rightarrow-\infty$ corresponds to

$$
\beta_{n}(t) \approx \mu_{a}(t)+2 \sigma_{a}^{-2}(t), \quad q_{\mathrm{opt}}^{(n)}(t) \approx q_{a}(t),
$$

where $\sigma_{a}^{2}(t)$ is the variance of the periodic distribution about the attractor given by Eq. (10).

To the lowest order in $q^{\prime}-q_{\mathrm{opt}}^{(n)}\left(t^{\prime}\right)$, the variance of the prehistory probability distribution (36) is also expressed in terms of the function $\beta_{n}$ [26],

$$
\sigma_{n}^{2}\left(t^{\prime}, t\right)=2 \int_{t}^{t^{\prime}} d \tau \kappa_{n}^{-2}(\tau, t)
$$

Using Eqs. (38), (40), and (41) one can show that $\sigma_{n}^{2}\left(t^{\prime}, t \rightarrow-\infty\right)=\sigma_{a}^{2}(t)$. For $t \rightarrow-\infty$ the distribution $p_{h}^{(n)}\left(q, t \mid q^{\prime}, t^{\prime}\right)$ goes over into the periodic Gaussian distribution $\rho_{a}(q, t)$, Eq. (8), centered at the periodic attractor.

It follows from Eqs. (36)-(39) that, for $q$ close to $q_{\mathrm{opt}}^{(n)}(t)$, the PPD $p_{h}^{(n)}\left(q, t \mid q^{\prime}, t^{\prime}\right)$ displays a diffusion-broadened Gaussian peak as a function of $q^{\prime}$ with maximum close to $q_{\mathrm{opt}}^{(n)}\left(t^{\prime}\right)$. The displacement of the maximum over $q^{\prime}$ from $q_{\mathrm{opt}}^{(n)}\left(t^{\prime}\right)$ is $\propto\left[q-q_{\mathrm{opt}}^{(n)}(t)\right]$. On the other hand, the function $p_{h}\left(q^{\prime}, t^{\prime} \mid q_{f}, t_{f}\right)$ as given by Eq. (32) is a sum of Gaussian distributions centered at the optimal escape paths. Therefore integration over $q^{\prime}$ in Eq. (35) can be done by the steepest descent method. The extreme values of $q^{\prime}$ are also close to the optimal escape paths.

Different peaks of the PPD $p_{h}\left(q^{\prime}, t^{\prime} \mid q_{f}, t_{f}\right)$, Eq. (32), correspond to the MPEP's shifted by an integer number of modulation periods. For each of these peaks one should use in Eq. (35) the PPD $p_{h}\left(q, t \mid q^{\prime} t^{\prime}\right)$ given by $p_{h}^{(n)}$ with the appropriate $n$.

The result of integration over $q^{\prime}$ describes the peaks of the PPD $p_{h}\left(q, t \mid q_{f}, t_{f}\right)$ for $q$ close to $q_{\mathrm{opt}}^{(n)}(t)$ with appropriate $n$. These peaks are Gaussian near the maxima, 


$$
p_{h}\left(q, t \mid q_{f}, t_{f}\right)=\frac{1}{\sum_{n} B_{n}\left(q_{f}, t_{f}\right)} \sum_{n} B_{n}\left(q_{f}, t_{f}\right) G\left(q-q_{\mathrm{opt}}^{(n)}(t)-\left(Q_{f}-Q_{\mathrm{opt}}^{(n)}\left(t_{f}\right)\right) \kappa_{n}^{-1}\left(t_{f}, t\right) ; \sigma_{n}\left(t_{f}, t\right)\right) .
$$

In obtaining this equation we used the relations

$$
\begin{aligned}
& \kappa_{b}\left(t_{f}, t^{\prime}\right) \kappa_{n}\left(t^{\prime}, t\right)=\kappa_{n}\left(t_{f}, t\right), \\
& \sigma_{n}^{2}\left(t^{\prime}, t\right)+\sigma_{f}^{2}\left(t_{f}, t^{\prime}\right) \kappa_{n}^{-2}\left(t^{\prime}, t\right)=\sigma_{n}^{2}\left(t_{f}, t\right),
\end{aligned}
$$

which in turn follow from the relation $\beta_{n}(t) \approx \mu_{b}(t)$ in the harmonic region near the basin boundary. As a consequence, the result of integration over $q^{\prime}$ in Eq. (35) is independent of the matching time $t^{\prime}$.

Equation (42) is the central result of the present paper. It gives, in the explicit form, the PPD of finding the system at a position $q$ inside the attraction basin at a moment $t$, provided the system has been observed at a point $q_{f}$ outside the attraction basin at a time $t_{f}>t$. It shows that, in the regime of strong synchronization, the peaks are Gaussian. They are centered at the most probable escape paths and are diffusion broadened throughout the attraction basin. This is in dramatic contrast with the PPD in the absence of synchronization, where the PPD peak inside the attraction basin is strongly asymmetric and its width is independent of the noise intensity $D$ [15].

It follows from Eq. (42) that the PPD may have multiple peaks inside the attraction basin. They can be observed only for strongly nonadiabatic modulation, where the Suzuki time is $t_{S} \gtrsim \tau_{F}$. In this case the system stays in the diffusion layer around the basin boundary $q_{b}(t)$ long enough to accumulate influxes from several periodically repeated most probable escape paths $q_{\mathrm{opt}}^{(n)}(t)$. On the other hand, the modulation period $\tau_{F}$ should not be too short, because the strong synchronization of escape would be lost. Since synchronization loss occurs for $t_{\mathrm{r}} \gg \tau_{F}$, the PPD displays well resolved multiple peaks only in a limited parameter range. The limitation is more restrictive to the considered case where the final point $\left(q_{f}, t_{f}\right)$ is close to the maximum of the distribution behind the barrier. If this condition is not imposed, the peaks are well resolved in a broader range, but measuring the PPD becomes more complicated on the whole. An example is discussed in the next Section.

An important feature of the distribution (42) is weak dependence of the shape of the Gaussian peaks inside the attraction basin on the final point $\left(q_{f}, t_{f}\right)$, which is a consequence of the smallness of the factor $\kappa^{-1}\left(t_{f}, t\right)$. This shows that the PPD reveals the actual structure of the tubes of the paths followed in escape inside the attraction basin. In contrast, the relative amplitudes of the PPD peaks $B_{n}$ are sensitive to the choice of the point $\left(q_{f}, t_{f}\right)$.

\section{RESULTS FOR A MODEL SYSTEM}

In this section, we present the results of numerical simulations of the PPD for a simple model system and compare them with the analytical predictions. We consider a Brownian particle in a sinusoidally modulated potential of the form of a cubic parabola. The Langevin equation of motion has the form of Eq. (1) with

$$
K(q, t)=q^{2}-\frac{1}{4}+A \cos \left(\omega_{F} t\right) .
$$

The dynamics was simulated using the second-order integration scheme for stochastic differential equations [27]. The system was initially prepared in the vicinity of the metastable state $q_{a}(t)$. The final point $\left(q_{f}, t_{f}\right)$ was chosen behind the basin boundary $q_{b}(t)$. The PPD was calculated as a normalized probability distribution of paths $q(t)$ arriving at the point $q_{f}$ for a particular modulation phase $\phi_{f}=\omega_{F} t_{f}(\bmod 2 \pi)$.

An example of the full PPD is shown in Fig. 2 The point $\left(q_{f}, t_{f}\right)$ is chosen so that in escape the system is likely to pass near it $\left(t_{f}\right.$ is determined $\left.\bmod \tau_{F}\right)$, that is the quasistationary distribution $\rho\left(q_{f}, t_{f}\right)$ is close to its maximum over $t_{f}$ for a given $q_{f}$ behind the basin boundary [the parameter $p_{\mathrm{opt}}^{\left(n_{0}\right)}\left(t_{f}\right)\left[q_{f}-q_{b}\left(t_{f}\right)\right] / D$ is equal to 1.2 , whereas the maximum of $\rho\left(q_{f}, t_{f}\right)$ is expected where this parameter is equal to 1, 201]. For the chosen modulation parameters and noise intensity the calculated activation energy of escape is $R \approx 0.0910$ and $R / D \approx 9.1$; the ratio of the modulation frequency to the relaxation rate $\Omega_{F} \approx 2.24$. We accumulated $\sim 10^{5}$ escape trajectories that arrive into a small area centered at $\left(q_{f}, t_{f}\right)$, with width $\delta q=0.02, \delta t=0.02 \tau_{F}$.

It is seen from Fig. 2 that, in the regime of strong synchronization and for strongly non-Gaussian pulses of escape current, the peaks of the PPD are narrow both behind the basin boundary and inside the attraction basin. Moreover, for the chosen parameter values two distinct peaks of the PPD are well resolved inside the attraction basin. They correspond to the two paths the system is most likely to follow in escape.

The positions of the PPD peaks on $(q, t)$ plane are shown in Fig. 4 with full squares where there is one peak, and with crosses where two peaks are well resolved. They are compared with the periodically repeated optimal escape paths calculated by solving numerically the variational equations (11) for the model (11), (43). Such paths start from the periodic attractor $q_{a}(t)$ for $t \rightarrow-\infty$ and approach the basin boundary $q_{b}(t)$ for $t \rightarrow \infty$, which are also shown in the figure. It is seen that, as expected from our analysis, the PPD maxima lie nearly on top of 


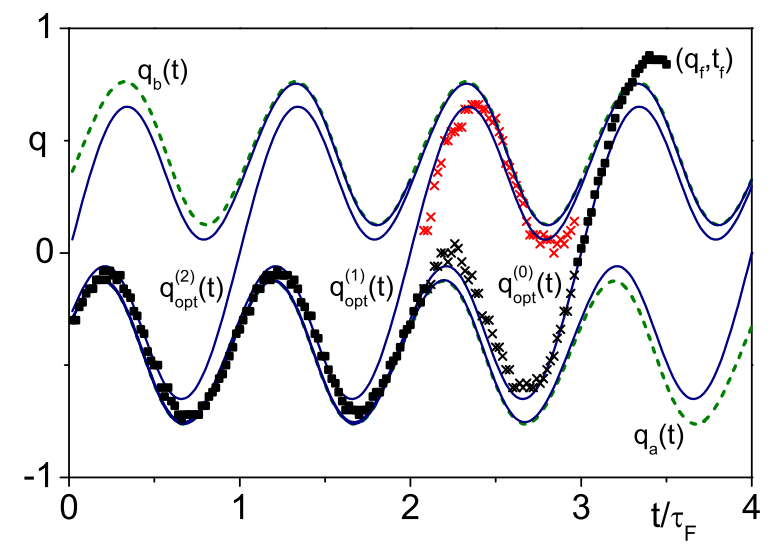

FIG. 4: (Color online) The positions of the maxima of the $\operatorname{PPD} p_{h}\left(q, t \mid q_{f}, t_{f}\right)$ in Fig. 2] which show the most probable paths followed by the system in escape. The data of simulations are shown by full squares where the PPD has one peak and by crosses where two peaks are well resolved. Solid lines show periodically repeated most probable escape paths $q_{\mathrm{opt}}^{(n)}(t)$ for the model (1), (43). Dashed lines show the basin boundary $q_{b}(t)$ and the attractor $q_{a}(t)$.

the most probable escape paths. The small deviation is due to diffusion broadening and associated small asymmetry of the PPD peaks for the noise intensity used in the simulations. Fig. 4 demonstrates that studying the PPD provides a direct way of observing most probable escape paths.

The observed shape of the PPD peaks is compared with the theory in Fig. [5] which shows the cross-sections of the PPD at several instants of time counted off from the final time $t_{f}$. For small $t_{f}-t$ the system is behind the basin boundary and moves close to the noise-free trajectory leading to $\left(q_{f}, t_{f}\right)$. The top left panel refers to the case where the system is localized close to the boundary. Here and for smaller $t_{f}-t$ the PPD has a single sharp peak.

For earlier time (larger $t_{f}-t$ ) the system could either be moving towards the basin boundary along the most probable escape path or could have been fluctuating about the basin boundary after it had arrived to its vicinity along the previous MPEP (shifted by $\tau_{F}$ ). As we showed analytically, the probability of staying near the boundary is smaller, but the PPD may still display two peaks. This is seen in the right top panel. The main peak corresponds to motion along the MPEP, which is close to $q_{b}(t)$ for the chosen time. The higher- $q$ shoulder corresponds to the poorly resolved (for the chosen time) peak for fluctuations about the basin boundary.

For still larger but not too large $t_{f}-t$ the escaping system should have been moving towards the basin boundary. In the present case it most likely followed one of the two periodically repeated MPEPs, with different probabilities. Well-resolved peaks of the PPD in this range are seen in the left lower panel of Fig. 5 For large $t_{f}-t$ compared to the relaxation time and $\tau_{F}$, the system should have been fluctuating about the attractor $q_{a}(t)$. The
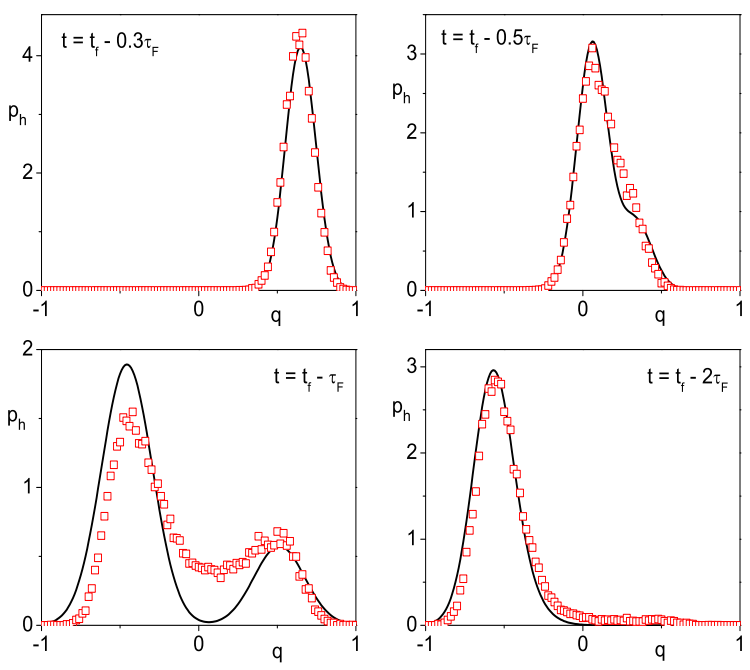

FIG. 5: (Color online) Cross-sections of the PPD $p_{h}\left(q, t \mid q_{f}, t_{f}\right)$ shown in Fig. 2 as functions of the coordinate $q$ for the time $t_{f}-t=0.3 \tau_{F}, 0.5 \tau_{F}, \tau_{F}, 2 \tau_{F}$. The results refer to the model system (11), (43) with $A=0.7, \omega_{F}=2$, $D=0.01, q_{f}=0.8, t_{f}=0.5 \tau_{F}$. The point $\left(q_{f}, t_{f}\right)$ is close to the expected maximum of the distribution behind the basin boundary. Solid lines show the expression (42). Squares show the results of simulations.

PPD in this case should have a single peak, which is seen in the right lower panel.

It is seen from Fig. 5 that the results of simulations agree with the analytical results. Not surprisingly, the observed peaks are broader than the asymptotic theory predicts. This is a consequence of the relatively large noise intensity used in the simulations.

\section{CONCLUSIONS}

In this paper we have studied the prehistory probability distribution for activated escape in periodically modulated systems. We have shown that the PPD as a function of coordinates can display one or several narrow peaks within the basin of attraction to the metastable state. They correspond to narrow ridges of the PPD in $(q, t)$ space. The ridges are centered at the periodically repeated most probable escape paths. Their crosssections are Gaussian near the maxima and are diffusion broadened, see Eq. (42).

The number of the PPD peaks that can be observed depends on the parameters of the system. The multi-peak structure is best resolved in a limited parameter range. On the one hand, the modulation should not be too slow, so that the system does not follow it adiabatically. On the other hand, it should not be too fast, so that escape events are strongly synchronized and the system dynamics is not described by period-averaged coordinates.

Most of the results of the paper refer to the case where the final point $\left(q_{f}, t_{f}\right)$ is chosen so that the escaped sys- 
tem has a high probability density of passing through this point. Such choice simplifies the experimental observation of the PPD. For the corresponding $\left(q_{f}, t_{f}\right)$ the amplitudes of different peaks of the PPD [heights of the ridges in $(q, t)$ space] differ from each other significantly, as seen from Eq. (34) and Figs. 2] 5] These amplitudes are sensitive to the choice of $\left(q_{f}, t_{f}\right)$. In contrast, the positions and shapes of the PPD peaks very weakly depend on $\left(q_{f}, t_{f}\right)$. This shows that the PPD provides a means for studying the distribution of trajectories leading to escape.

We have performed extensive numerical simulations of escape for a model Brownian particle in a modulated potential well. The simulations confirm the possibility to clearly observe most probable escape paths. They are in a good qualitative and quantitative agreement with the analytical theory.

Observing most probable escape paths is not only interesting on its own, but also has broader implications. First, periodically modulated systems are an important class of systems far from thermal equilibrium. In contrast to the case of equilibrium systems, optimal fluctuational paths in nonequilibrium systems have no immediate re- lation to dynamical trajectories in the absence of noise; in particular, they may not be obtained by just reversing time. The MPEP's can display interesting and counterintuitive behavior [21, 28], and studying them provides an insight into general features of dynamics away from thermal equilibrium. Second, understanding the dynamics of a system in escape paves the way for efficient control of this dynamics and the escape probability itself; as will be discussed in a separate publication, such control can be accomplished by comparatively weak field pulses applied in the right place and at the right time.

In conclusion, the results of this paper suggest a way of direct observation of most probable escape paths, in space and time. They also describe, qualitatively and quantitatively, the distribution of the trajectories followed in escape.

\section{ACKNOWLEDGMENTS}

This research has been supported in part by the NSF Grant No. DMR-0305746.
[1] M. H. Devoret, D. Esteve, J. M. Martinis, A. Cleland, and J. Clarke, Phys. Rev. B 36, 58 (1987).

[2] E. Turlot, S. Linkwitz, D. Esteve, C. Urbina, M. H. Devoret, and H. Grabert, Chem. Phys. 235, 47 (1998).

[3] I. Siddiqi, R. Vijay, F. Pierre, C. M. Wilson, L. Frunzio, M. Metcalfe, C. Rigetti, R. J. Schoelkopf, M. H. Devoret, D. Vion, and D. Esteve, Phys. Rev. Lett. 94, 027005 (2005).

[4] J. S. Aldridge and A. N. Cleland, Phys. Rev. Lett. 94, 156403 (2005).

[5] C. Stambaugh and H. B. Chan, cond-mat/0504791 (2005).

[6] I. B. Schwartz, L. Billings, and E. M. Bollt, Phys. Rev. E 70, 046220 (2004).

[7] D. Moroni, T. S. van Erp, and P. G. Bolhuis, Physica A 340, 395 (2004).

[8] D. M. Zuckerman, J. Phys. Chem. B 108, 5127 (2004).

[9] W. N. E, W. Q. Ren, and E. Vanden-Eijnden, Phys. Rev. B 66, 052301 (2002).

[10] S. M. Soskin, R. Mannella, and P. V. E. McClintock, Phys. Rep. 373, 247 (2003).

[11] M. I. Dykman, P. V. E. McClintock, V. N. Smelyanski, N. D. Stein, and N. G. Stocks, Phys. Rev. Lett. 68, 2718 (1992).

[12] D. G. Luchinsky and P. V. E. McClintock, Nature 389, 463 (1997).

[13] M. Morillo, J. M. Casado, and J. GomezOrdonez, Phys. Rev. E 55, 1521 (1997).
[14] M. Arrayas, J. M. Casado, J. G. Ordonez, P. V. E. McClintock, M. Morillo, and N. D. Stein, Phys. Rev. Lett. 80, 2273 (1998).

[15] J. Hales, A. Zhukov, R. Roy, and M. I. Dykman, Phys. Rev. Lett. 85, 78 (2000).

[16] V. N. Smelyanskiy, M. I. Dykman, H. Rabitz, and B. E. Vugmeister, Phys. Rev. Lett. 79, 3113 (1997).

[17] J. Lehmann, P. Reimann, and P. Hanggi, Phys. Rev. Lett. 84, 1639 (2000).

[18] R. S. Maier and D. L. Stein, Phys. Rev. Lett. 86, 3942 (2001)

[19] M. I. Dykman and D. Ryvkine, Phys. Rev. Lett. 94, 070602 (2005).

[20] D. Ryvkine and M. I. Dykman, Phys. Rev. E 72, 011110 (2005).

[21] M. I. Dykman, Phys. Rev. A 42, 2020 (1990).

[22] M. I. Freidlin and A. D. Wentzell, Random perturbations of dynamical systems (Springer-Verlag, New York, 1998), 2nd ed.

[23] R. Graham and T. Tel, Phys. Rev. Lett. 52, 9 (1984).

[24] M. Suzuki, J. Stat. Phys. 16, 11 (1977).

[25] M. Suzuki, J. Stat. Phys. 16, 477 (1977).

[26] M. I. Dykman, D. G. Luchinsky, P. V. E. McClintock, and V. N. Smelyanskiy, Phys. Rev. Lett. 77, 5229 (1996).

[27] R. Mannella, Intl. J. Mod. Phys. C 13, 1177 (2002).

[28] R. S. Maier and D. L. Stein, Phys. Rev. Lett. 71, 1783 (1993). 